\title{
SPECTROSCOPY ALONG MULTIPLE, LENSED SIGHT LINES THROUGH OUTFLOWING WINDS IN THE QUASAR SDSS J1029+2623*
}

\author{
Toru Misawa ${ }^{1}$, Naohisa Inada ${ }^{2}$, Ken Ohsuga $^{3,4}$, Poshak Gandhi $^{5}$, Rohta Takahashi $^{6}$, And Masamune Oguri ${ }^{7}$ \\ ${ }^{1}$ School of General Education, Shinshu University, 3-1-1 Asahi, Matsumoto, Nagano 390-8621, Japan; misawatr@ shinshu-u.ac.jp \\ ${ }^{2}$ Department of Physics, Nara National College of Technology, Yamatokohriyama, Nara 639-1080, Japan \\ ${ }^{3}$ National Astronomical Observatory of Japan, Osawa, Mitaka, Tokyo 181-8588, Japan \\ ${ }^{4}$ School of Physical Sciences, Graduate University of Advanced Study (SOKENDAI), Shonan Village, Hayama, Kanagawa 240-0193, Japan \\ ${ }^{5}$ Institute of Space and Astronautical Science (ISAS), Japan Aerospace Exploration Agency, 3-1-1 Yoshinodai, chuo-ku, Sagamihara, Kanagawa 252-5210, Japan \\ ${ }^{6}$ Department of Natural and Physical Sciences, Tomakomai National College of Technology, Tomakomai 059-1275, Japan \\ ${ }^{7}$ Kavli Institute for the Physics and Mathematics of the Universe (Kavli IPMU, WPI), University of Tokyo, Chiba 277-8583, Japan \\ Received 2012 October 3; accepted 2012 December 11; published 2013 January 15
}

\begin{abstract}
We study the origin of absorption features on the blue side of the C IV broad emission line of the large-separation lensed quasar SDSS J1029+2623 at $z_{\mathrm{em}} \sim 2.197$. The quasar images, produced by a foreground cluster of galaxies, have a maximum separation angle of $\theta \sim 22$ '.5. The large angular separation suggests that the sight lines to the quasar central source can go through different regions of outflowing winds from the accretion disk of the quasar, providing a unique opportunity to study the structure of outflows from the accretion disk, a key ingredient for the evolution of quasars as well as for galaxy formation and evolution. Based on medium- and high-resolution spectroscopy of the two brightest images conducted at the Subaru telescope, we find that each image has different intrinsic levels of absorptions, which can be attributed either to variability of absorption features over the time delay between the lensed images, $\Delta t \sim 744$ days, or to the fine structure of quasar outflows probed by the multiple sight lines toward the quasar. While both these scenarios are consistent with the current data, we argue that they can be distinguished with additional spectroscopic monitoring observations.
\end{abstract}

Key words: quasars: absorption lines - quasars: individual (SDSS J1029+2623)

Online-only material: color figures

\section{INTRODUCTION}

Quasars are routinely used as background sources to study the gaseous phases of intervening objects via absorption-line diagnostics. These absorption lines have their origins both in intervening objects (i.e., foreground galaxies, intergalactic medium (IGM), quasar host galaxies) and in sources that are intrinsic to the quasars. One of the most promising candidates for the intrinsic absorbers is an outflowing wind from the accretion disk of quasar central engines. The outflow is accelerated by magnetocentrifugal forces (Everett 2005; de Kool \& Begelman 1995), radiation pressure in lines and continuum (Murray et al. 1995; Proga et al. 2000), and/or by thermal pressure force (e.g., Balsara \& Krolik 1993; Krolik \& Kriss 2001; Chelouche \& Netzer 2005). The outflowing winds play an important role in three ways: (1) the extraction of angular momenta from disks allows accretions to proceed (e.g., Blandford \& Payne 1982; Emmering et al. 1992; Konigl \& Kartje 1994; Everett 2005), leading to growth of black holes (BHs); (2) the disk outflow also provides energy and momentum feedback to interstellar media (ISM) of host galaxies and to IGM, and inhibits star formation activity (e.g., Springel et al. 2005); (3) outflowing winds may induce the metal enrichment of the IGM (e.g., Hamann et al. 1997a; Gabel et al. 2006). Thus, the physical conditions of the outflow not only promote the evolution of quasars themselves, but greatly impact the surrounding environments.

Among absorption lines, broad features (hereafter BALs; FWHM $\geqslant 2000 \mathrm{~km} \mathrm{~s}^{-1}$ ) are easily identified as being intrinsic because it is almost impossible for foreground objects to produce

\footnotetext{
* Based on data collected at Subaru Telescope, which is operated by the National Astronomical Observatory of Japan.
}

very broad and smooth line profiles. BALs are detected in about $10 \%-20 \% 8$ of optically selected quasars (e.g., Hewett \& Foltz 2003; Reichard et al. 2003), and their detection rate is slightly higher in radio-quiet quasars (e.g., Stocke et al. 1992; Becker et al. 2001; Green 2006). BALs are thought to originate in the outflowing winds when our sight line intersects this component. This idea is supported by the fact that there are no significant differences in the properties of quasars with BALs (BAL QSOs) and those without BALs (non-BAL QSOs; Weymann et al. 1991; Reichard et al. 2003). Moreover, quasar spectra tend to be redder when BAL profiles, especially those with low ionization absorption lines (i.e., LoBALs and FeLoBALs), are observed, which is probably caused by dust reddening in the outflows (e.g., Sprayberry \& Foltz 1992; Yamamoto \& Vansevičius 1999). Thus, quasars with and without BALs may intrinsically be a single population, although the evolutionary phase of quasars could also affect the detection rate of BALs (e.g., Voit et al. 1993). The only difference is whether or not the sight line passes through the outflowing wind in this orientation scheme. Thus, BAL QSOs have traditionally been the best targets for the study of outflows (e.g., Weymann et al. 1991; Becker et al. 1997; Gibson et al. 2009; Capellupo et al. 2012).

In addition to BALs, a fraction of narrow absorption lines (hereafter NALs; FWHM $\leqslant 500 \mathrm{~km} \mathrm{~s}^{-1}$ ) and mini-BALs (an intermediate category between BALs and NALs) have also been suggested to be physically associated with quasars. However, the origin of NALs and mini-BALs are still under debate. Each of the above classes of absorption features could represent either

\footnotetext{
8 However, we may underestimate the detection rate of BALs based on flux-limited optical surveys because Chartas (2000) found that approximately $35 \%$ of radio-quiet gravitational lensed quasars contain BAL features.
} 


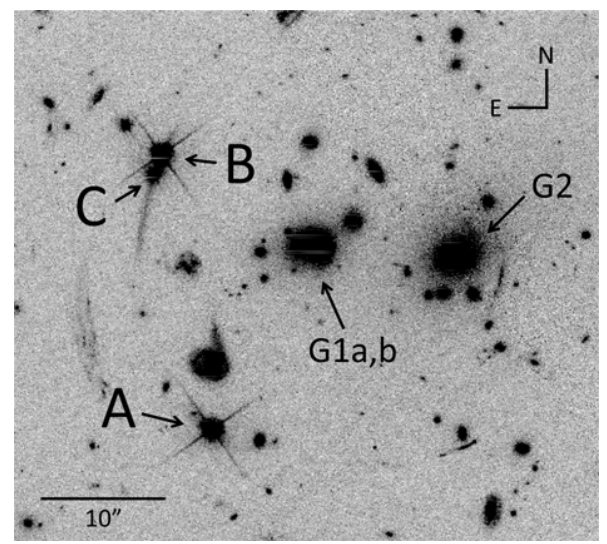

Figure 1. F814W-band image of SDSS J1029+2623 taken with HST/ACS (GO-12195; Oguri et al. 2012). Three objects labeled with A, B, and C are lensed images of the quasar at $z_{\mathrm{em}}=2.197$, while those with G1a, G1b, G2 are member galaxies of a foreground lensing cluster at $z \sim 0.6$. Angular separations of the images are $\theta_{\mathrm{AB}} \sim 22^{\prime \prime} .5, \theta_{\mathrm{AC}} \sim 21^{\prime \prime}$. 0 , and $\theta_{\mathrm{BC}} \sim 1^{\prime \prime} .85$.

different lines of sight through the outflowing wind to the quasar continuum source or different stages in the evolution of the absorbing gas parcels (e.g., Hamann \& Sabra 2004; Misawa et al. 2005). The observed fraction of optically selected quasars hosting BALs $(\sim 10-20)$ and NALs $(\sim 20 \%-50 \%$, e.g., Vestergaard 2003; Wise et al. 2004; Misawa et al. 2007a; Nestor et al. 2008) constrains the solid angle subtended by the dense portion of the wind to the central engine. However, such a statistical treatment requires the assumption that all quasars are identical. This remains an assumption because in past studies it has only been possible to trace single sight lines (i.e., probe a one-dimensional profile) toward the nucleus of each quasar.

Gravitationally lensed images of background quasars are our only tools for the study of the three-dimensional geometry of the absorbers (e.g., Crotts \& Fang 1998; Rauch et al. 1999; Lopez et al. 2005). Indeed, this technique has frequently been applied for investigating intervening absorption lines, produced by galaxies or IGM on scales of up to several kiloparsecs. However, these traditional quasar lenses are not useful for multiangle studies of the active galactic nuclei themselves because their separation angles $(\theta \sim$ a few arcsec) are too small to separate internal structures in the vicinity of the quasars. On the other hand, Inada et al. (2003) discovered a lensed quasar, SDSS J1004+4112, with a maximum image separation of $\theta \sim 14$.' 6 , which is the first example of a quasar lensed by a cluster of galaxies rather than a single massive galaxy. If there exists outflow gas at a distance of $\sim 1 \mathrm{kpc}^{9}$ from the continuum source as measured for some specific quasars (de Kool et al. 2001; Hamann et al. 2001), the large separation angle of SDSS J1004+4112 translates into a physical distance of $\gtrsim 0.1 \mathrm{pc}$, with which we may trace outflowing winds with different physical properties unless their transverse sizes are much larger than subparsec scale. Taking advantage of this large image separation, Green (2006) proposed that the differences in emission lines between the lensed images seen in their follow-up spectra can be explained by differential absorptions along each sight line, although the spectra do not show explicit intrinsic absorption features.

The second large-separation lensed quasar SDSS J1029+2623 (Inada et al. 2006) has an image separation of $\theta \sim 22$.'5, and

\footnotetext{
9 However, we usually have only loose constraints on the absorber's distance,
} spanning from $r=0.01$ to 1000 pc (e.g., Elvis 2000; de Kool et al. 2001).

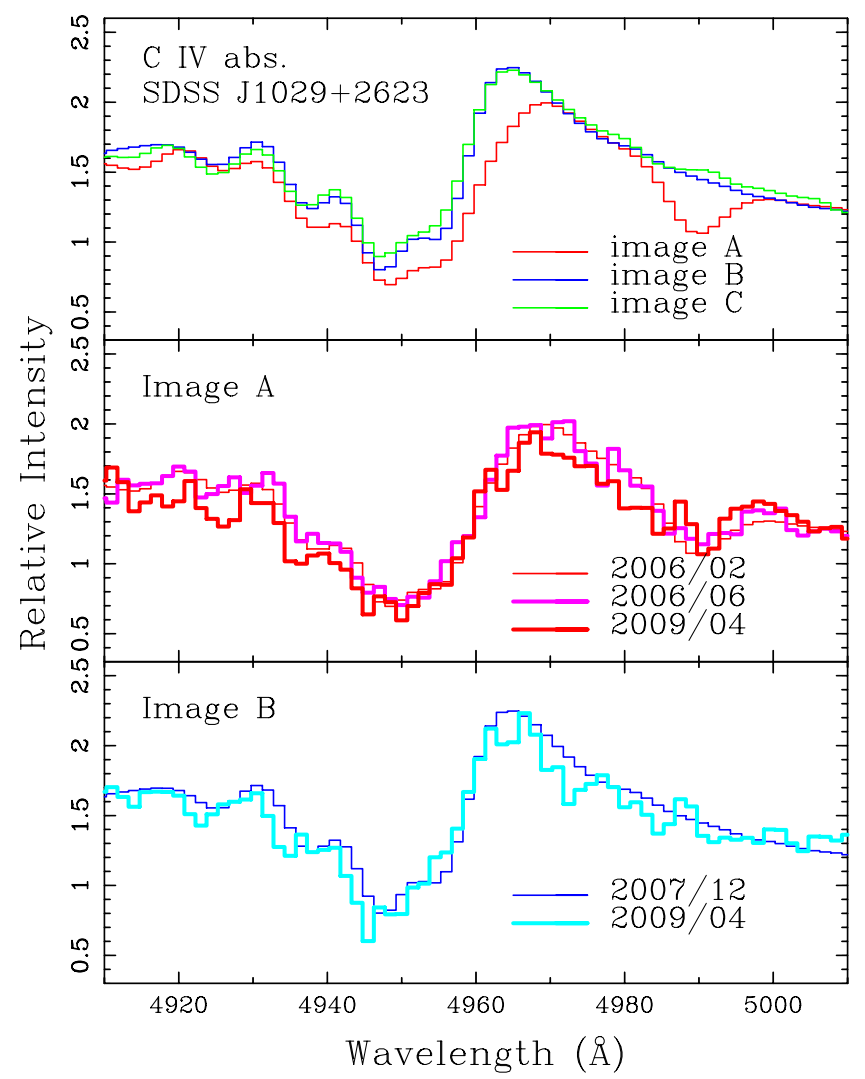

Figure 2. Medium-resolution spectra $(R \sim 500)$. Top: zoomed spectra of images A, B, and C of SDSS J1029+2623 around the associated C IV absorption line (Inada et al. 2006; Oguri et al. 2008). Fluxes are arbitrary and normalized to compare the absorption profiles of the three images. An additional absorption line at $\sim 4990 \AA$ present only in the image A spectrum is Fe II $\lambda 2600$ at $z_{\text {abs }} \sim 0.9187$. Middle: spectra of image A, taken in 2006 twice (Inada et al. 2006; York et al. 2000) and in 2009 by us. Bottom: same as the above panel, but for image B, taken in 2007 (Oguri et al. 2008) and in 2009 by us.

(A color version of this figure is available in the online journal.)

therefore is the largest quasar lens currently known. Oguri et al. (2008) identified the third image of this lens system (see Figure 1). As shown in Figure 2, all the spectra of quasar images $\mathrm{A}, \mathrm{B}$, and $\mathrm{C}$ have absorption features on the blue side of the C IV emission lines, which implies that each sight line passes through the outflowing wind. In what follows, we call this feature "the associated absorption line." ${ }^{10}$ Moreover, we see a clear difference in the absorption features between image A and images B/C. The former has a broader and shallower profile and an additional redshifted absorption line at $\sim 4990 \AA$, while the latter two have narrower and deeper profiles (Figure 2). After correcting for magnification, the Eddington ratio of the quasar is estimated to be $L / L_{E} \sim 0.11$. This value is consistent with the typical Eddington ratio of bright quasars, $L / L_{E} \sim 0.07-1.6$ (Netzer et al. 2007).

In this paper, we discuss the origin of these differential absorption profiles in SDSS J1029+2623. The likely scenarios include (1) time variation of a single absorber covering both sight lines toward the quasar between the time delay of images $\mathrm{A}$ and $\mathrm{B} / \mathrm{C}$, which is $\sim 744$ days in the observed frame according

\footnotetext{
10 A term associated absorption line is traditionally used for absorption features that fall within $5000 \mathrm{~km} \mathrm{~s}^{-1}$ of quasar emission redshift (Weymann et al. 1979). We use this term for this specific absorption feature throughout the
} paper. 
Table 1

Log of Observations

\begin{tabular}{lccccccc}
\hline \hline Target & Date & Order $^{\mathrm{a}}$ & Instrument & $R$ & $\begin{array}{c}\text { Exp. } \\
(\mathrm{s})\end{array}$ & $\begin{array}{c}\mathrm{S}^{\mathrm{N}} \mathrm{N}^{\mathrm{b}} \\
\left(\mathrm{pixel}^{-1}\right)\end{array}$ & Reference $^{\mathrm{c}}$ \\
$(1)$ & $(2)$ & $(3)$ & $(4)$ & $(5)$ & $(6)$ & $(7)$ & $(8)$ \\
\hline Image A & 2006 Feb 28 & 2 & SDSS & 1800 & & 9.1 & 1 \\
& 2006 Jun 29 & 3 & Subaru/FOCAS & 500 & 600 & 19 & 2 \\
& 2009 Apr 4 & 5 & Subaru/FOCAS & 500 & 1200 & 8.5 & 3 \\
Image B & 2007 Dec 15 & 1 & Keck/LRIS & 1000 & 800 & 43 & 4 \\
& 2009 Apr 4 & 4 & Subaru/FOCAS & 500 & 1200 & 7.0 & 3 \\
Image C & 2007 Dec 15 & 1 & Keck/LRIS & 1000 & 800 & 11 & 4 \\
\hline Image A & 2010 Feb 10 & & Subaru/HDS & 30000 & 14400 & 13 & 3 \\
Image B & 2010 Feb 10 & & Subaru/HDS & 30000 & 14200 & 13 & 3 \\
\hline
\end{tabular}

Notes.

${ }^{\text {a }}$ Order of observing epoch considering time delay, i.e., adding $\sim 744$ days only to the observation of image A.

b Signal-to-noise ratio around $4700 \AA$.

${ }^{c}$ References: (1) Sloan Digital Sky Survey York et al. 2000; (2) Inada et al. 2006; (3) this paper; (4) Oguri et al. 2008.

to monitoring observations by Fohlmeister et al. (2012) ${ }^{11}$ (hereafter scenario I); (2) each sight line penetrating different absorbers or different regions of a single absorber toward the quasar as proposed by Green (2006; scenario II); and (3) microlensing (scenario III). Among these, the last scenario has already been rejected because each image shows common ratios between radio, optical, and X-ray fluxes (Ota et al. 2012; Oguri et al. 2012), which is not expected for microlensing. Thus we concentrate on the viability of scenarios I and II, based on our medium- and high-resolution spectroscopic observations of SDSS J1029+2623.

The structure of this paper is as follows. In Section 2, we describe the observations and data reduction. We present results in Section 3, and discuss the results in Section 4. We summarize our results in Section 5. We adopt $z_{\mathrm{em}}=2.197$ as the emission redshift of the quasar, which was estimated using broad UV emission lines by Inada et al. (2006). Time intervals between observations are given in the observed frame throughout the paper, unless otherwise noted.

\section{OBSERVATIONS AND DATA REDUCTION}

We conducted spectroscopic observation of the two brightest lensed images $^{12}$ (i.e., images A and B) of SDSS J1029+2623 with Subaru/FOCAS on 2009 April 4, using a similar resolution power to previous observations $(R \sim 500)$ taken about $2-3$ years before. This time separation is comparable to the measured time delay between image $\mathrm{A}$ and images B/C ( 744 days; Fohlmeister et al. 2012). We used the grating with L600 filter to cover the wavelength range of $\lambda=3700-6000 \AA$. We also used the 0.4 slit without pixel sampling $\left(\sim 1.34 \AA\right.$ pixel $\left.^{-1}\right)$, which enables us to compare our spectra with the previous observations from the literature (Inada et al. 2006; Oguri et al. 2008) including SDSS (York et al. 2000) by performing convolution where needed. The sky conditions were moderate although affected by thin clouds. The total integration time was $1200 \mathrm{~s}$ for each image, and the data quality of the final spectra are $\mathrm{S} / \mathrm{N} \sim 8.5 \mathrm{pixel}^{-1}$ and 7.0 pixel $^{-1}$ for images $A$ and $B$, respectively.

We also obtained high-resolution spectra of images $A$ and $B$ with Subaru/HDS on 2010 February 10 to resolve the associated

\footnotetext{
11 The time delay between images $\mathrm{B}$ and $\mathrm{C}$ is almost negligible, $\sim 2-3$ days (Fohlmeister et al. 2012).

12 The observed flux ratio of three lensed images is A:B:C 0.95:1.00:0.24 in the $I$ band (Oguri et al. 2008).
}

C IV absorption line completely and extract physical parameters such as column density, line width, ejection velocity from the quasar, and the covering factor (the fraction of flux from the background source that is geometrically covered by the foreground absorber) using Voigt profile fitting. We used the standard setup, Std-Bc, with a 1".2 slit width $(R=30,000)$, covering the wavelength range of 3390-4210 ̊ on the blue CCD and $4280-5110 \AA$ on the red CCD. This configuration covers Ly $\alpha, \mathrm{N}$ v, Si IV, as well as the C IV absorption line at $z_{\text {abs }} \sim z_{\mathrm{em}}$. The CCD is binned every 4 pixels in both spatial and dispersion directions (i.e., $\sim 0.05 \AA$ pixel $^{-1}$ and each resolution element contains three pixels around $4500 \AA$ ). The total integration times were $14,400 \mathrm{~s}$ and $14,200 \mathrm{~s}$ for images $\mathrm{A}$ and $\mathrm{B}$, respectively, and the final signal-to-noise ratios $(\mathrm{S} / \mathrm{Ns})$ are about $13 \mathrm{pixel}^{-1}$ for both the images.

We reduced both the FOCAS and HDS data in a standard manner with the software IRAF. ${ }^{13}$ As for the HDS spectra, we divided them by a FLAT frame before normalization in order to remove the instrumental blaze pattern. Wavelength calibration was performed using a Th-Ar lamp. The observation log is summarized in Table 1.

\section{RESULTS}

\subsection{Low-resolution Spectroscopy}

We examined the suite of Subaru/FOCAS spectra to search for variability in the $\mathrm{C}$ IV lines. If absorption in both the images show time variations, it would imply that the profiles are variable and that the scenario I is more favorable. If this is the case, we have monitored a single absorber in five epochs on 2006 February $28+744$ days, 2006 June 29 + 744 days, 2009 April $4+$ 744 days (toward image A), and on 2007 December 15, 2009 April 4 (toward image B). If neither images show time variation, the scenario II will instead be favored.

\subsubsection{Image A}

The associated C IV absorption line of the image A was spectroscopically observed three times on 2006 February 28 (York et al. 2000), on 2006 June 29 (Inada et al. 2006), and on 2009 April 4 (this paper), as shown in Figure 2. The first

\footnotetext{
13 IRAF is distributed by the National Optical Astronomy Observatory, which is operated by the Association of Universities for Research in Astronomy, Inc. under cooperative agreement with the National Science Foundation.
} 

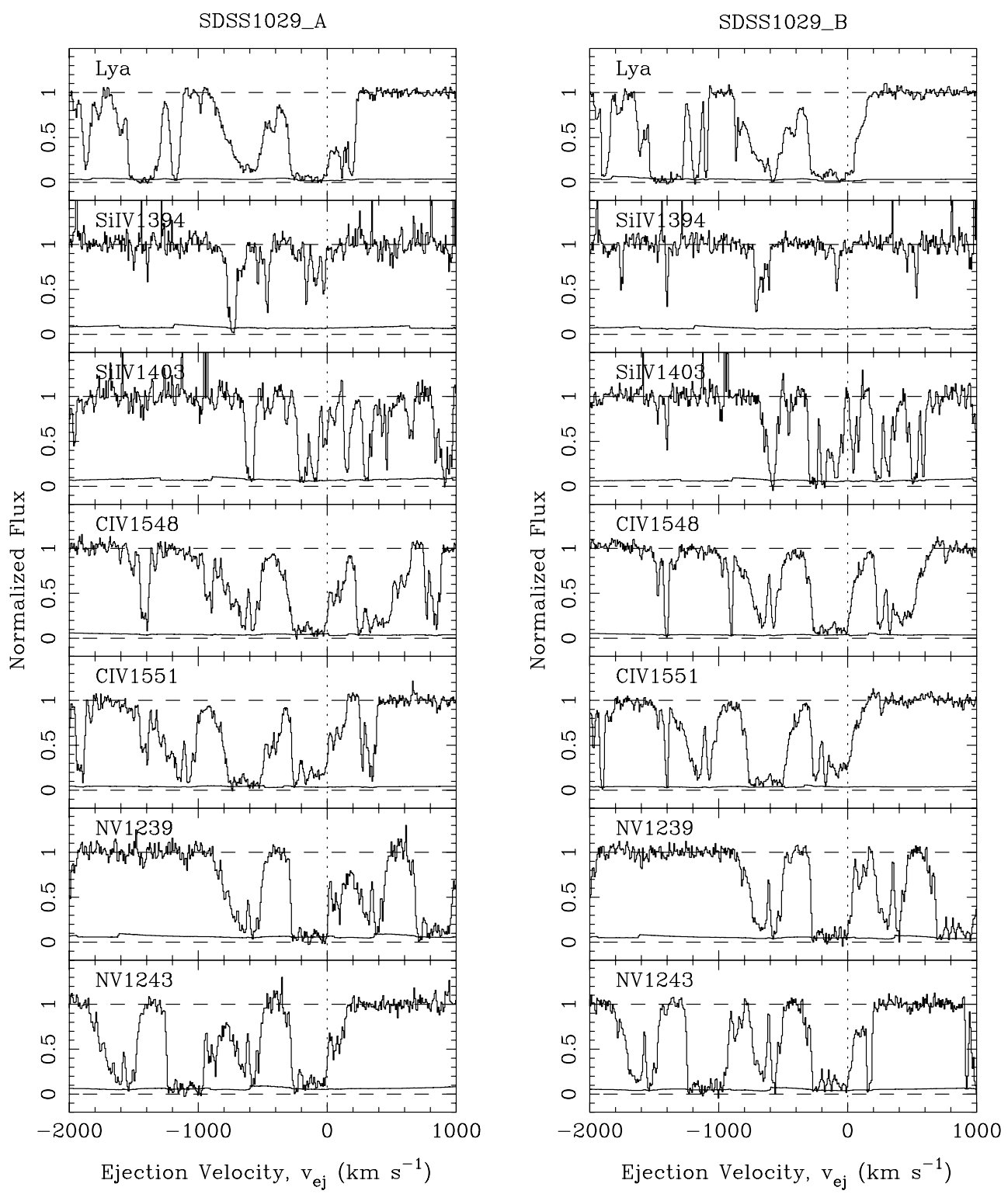

Figure 3. Velocity plot of the associated absorption system toward image A (left) and image B (right) with high-resolution spectra. Vertical dotted lines correspond to the emission redshift of the quasar, $z_{\mathrm{em}}=2.197$.

one was part of the Sloan Digital Sky Survey (SDSS), while the others were taken with Subaru/FOCAS. For inter-comparison, we adjust the spectral resolution of all observations to that lowest available (i.e., $R \sim 500$ ) by resampling and convolution. The $\mathrm{C}$ IV emission and absorption features show a so-called P-Cygni profile, which implies that the absorption feature is physically associated to the quasar itself. An additional weak absorption feature is seen on the red side of the C IV emission line at $\lambda \sim 4990 \AA$. However, this is not C IV, but Fe II $\lambda 2600$ at $z_{\text {abs }} \sim 0.9187$, because there exists a corresponding $\mathrm{Mg}$ II doublet at the same redshift. We do not see any substantial variation of the $\mathrm{C}$ IV absorption profile (see Figure 2).

\subsubsection{Image $B$}

The associated C IV absorption line of image B was spectroscopically observed only twice: once on 2007 December 15 with Keck/LRIS by Oguri et al. (2008), and once on 2009 April 4 by us with Subaru/FOCAS. Oguri et al. (2008) also obtained a spectrum of image $\mathrm{C}$. Images $\mathrm{B}$ and $\mathrm{C}$ show profiles that are almost identical near the $\mathrm{C}$ IV absorption/emission lines, which is probably because the separation angle between them, $\theta_{\mathrm{BC}} \sim$ 1 .' 85 , is much smaller than the separations between images $\mathrm{A}$ and $\mathrm{B}\left(\theta_{\mathrm{AB}} \sim 22^{\prime \prime} .5\right)$ and images $\mathrm{A}$ and $\mathrm{C}\left(\theta_{\mathrm{AC}} \sim 21^{\prime \prime}\right.$. 0$)$. Our FOCAS spectrum of image $B$ is consistent with those of images $\mathrm{B}$ and $\mathrm{C}$ taken about 15 months ( $\sim 4.7$ month in the rest frame) before.

Under scenario I, time variation is to be expected. Instead, we confirm that the absorption profile of each image is stable (see Figure 2), suggesting that scenario I is less likely to be true. However, this conclusion based on our medium-resolution spectroscopy will have to be modified significantly, following investigation of the high-resolution spectra, as we will see in the next subsection.

\subsection{High-resolution Spectroscopy}

From our high-resolution $(R=30,000)$ spectra of images A and $\mathrm{B}$, we find that the associated $\mathrm{C}$ IV absorption line consists of multiple narrower components (Figure 3). Thus, the system 

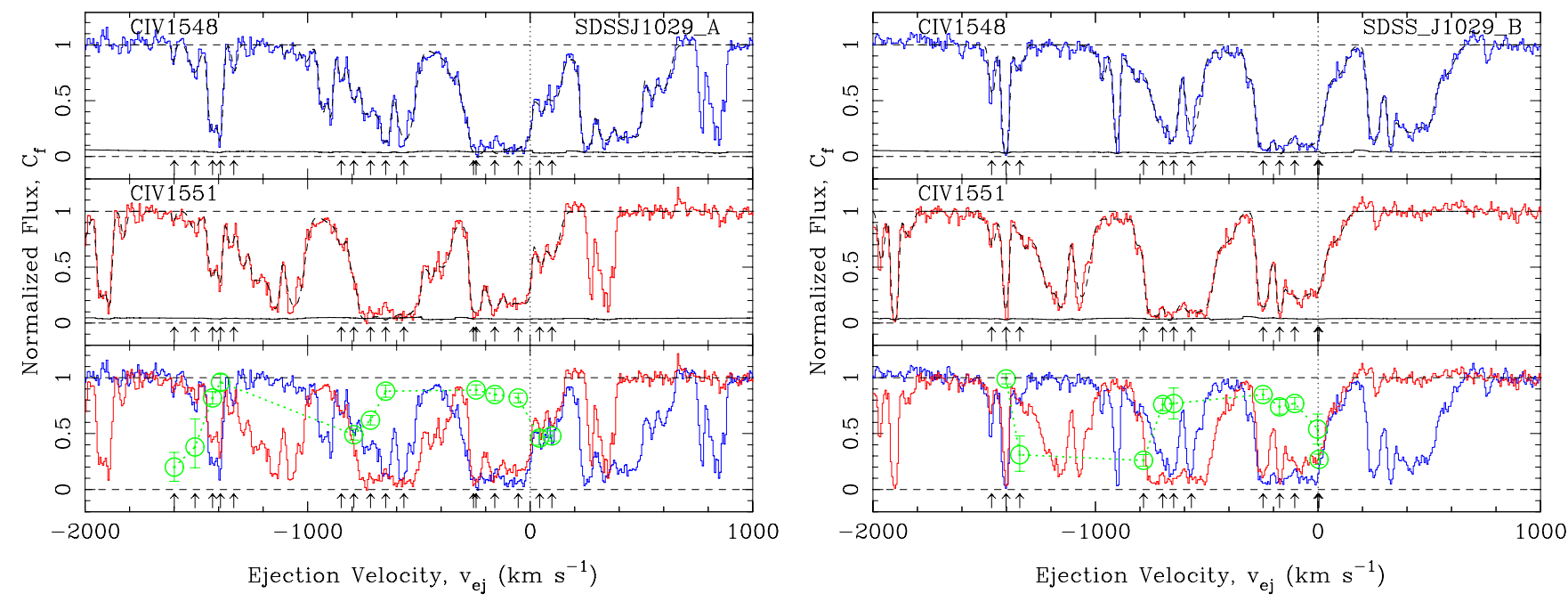

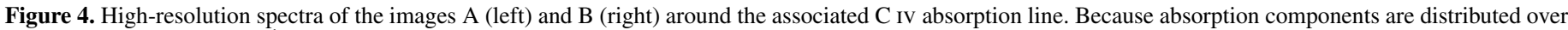

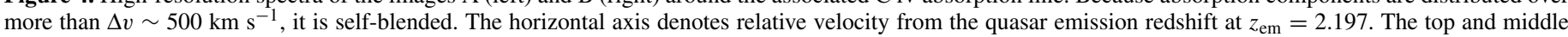

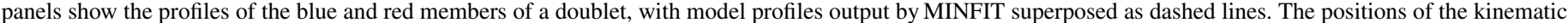

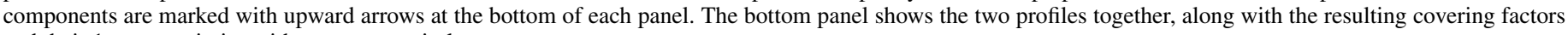
and their $1 \sigma$ uncertainties with green open circles.

(A color version of this figure is available in the online journal.)

is not a BAL (or a mini-BAL) but NAL, which means that the features are not necessarily arising at the outflow. If the corresponding absorber is the ISM of the host galaxy, IGM, or a foreground galaxy (i.e., intervening absorption), both the differential absorption profiles and the lack of time variation toward images A and B are reasonable because their sight lines pass through completely different regions separated by $\sim \mathrm{kpc}$ or $\sim \mathrm{Mpc}$ scales with respect to each other.

In order to classify the associated C IV absorption feature as an intrinsic or intervening feature, we performed a Voigt profile fitting to measure line parameters. We used the line fitting software package MINFIT (Churchill 1997; Churchill et al. 2003), with which we can fit absorption profiles using redshift $(z)$, column density $\left(\log N\right.$ in $\left.\mathrm{cm}^{-2}\right)$, Doppler parameter $(b$ in $\left.\mathrm{km} \mathrm{s}^{-1}\right)$, and covering factor $\left(C_{f}\right.$, defined later in Section 3.2.1) as free parameters. The revised version of the code can be applied to self-blending features, ${ }^{14}$ because it fits profiles to the blue and red members of doublets simultaneously, by multiplying the contributions from the two doublet members (Misawa et al. 2007b).

The fitting results are summarized in Table 2. Column 1 is a line identification (ID) number. Columns 2 and 3 give the absorption redshift and the ejection velocity (negative corresponds to blueshifted) from the quasar emission redshift, $z_{\mathrm{em}} \sim 2.197$. Columns 4 and 5 are the column density and its $1 \sigma$ error, Columns 6 and 7, the Doppler parameter and its $1 \sigma$ error, and Columns 8-10, the covering factors and its upper/lower $1 \sigma$ error. We also applied line fitting to the $\mathrm{N} \mathrm{v}$ doublet. The $\mathrm{Si}$ IV doublet cannot be fitted in both the images because the red member of the doublet, Si IV $\lambda 1403$, is severely affected by line contamination.

\subsubsection{Covering Factor Analysis}

Covering factor, $C_{f}$, is the fraction of coverage of the absorber over the continuum source and the broad emission line region

\footnotetext{
${ }^{14}$ Blue and red members of doublets such as $\mathrm{C}$ IV, $\mathrm{N}$ v, and Si IV are blended with each other if their line widths are greater than the velocity distance between the two members (e.g., $\Delta v \sim 500 \mathrm{~km} \mathrm{~s}^{-1}$ in the case of $\mathrm{C}$ IV doublet).
}

(BELR) along the sight line. The covering factor can be systematically evaluated in an unbiased manner by considering the optical depth ratio of resonant, rest-frame UV doublets of lithium-like species (e.g., $\mathrm{C}$ IV, $\mathrm{N} \mathrm{v}$, and $\mathrm{Si}$ IV), using the equation

$$
C_{f}=\frac{\left(R_{r}-1\right)^{2}}{1+R_{b}-2 R_{r}},
$$

where $R_{b}$ and $R_{r}$ are the residual (i.e., unabsorbed) fluxes of the blue and red members of the doublets in the continuum normalized spectrum (e.g., Hamann et al. 1997a; Barlow \& Sargent 1997; Crenshaw et al. 1999). A $C_{f}$ value less than unity indicates that a portion of the background source is not occulted by the absorber. This, in turn, means that the doublet is probably produced by an intrinsic absorber because intervening absorbers like IGM, ISM, and foreground galaxies usually have physical scales with a few kiloparsecs much larger than the background sources (e.g., Wampler et al. 1995; Barlow \& Sargent 1997). In general, the continuum source and the BELR have different covering factors, as discussed in Ganguly et al. (1999). The derived coverage fractions can be significantly affected by uncertainty of the continuum level, providing unphysical values (i.e., $C_{f}<0$ or $1<C_{f}$ ), especially for very weak components whose $C_{f}$ values are close to 1 . Therefore, if MINFIT gives unphysical values of $C_{f}$ for some components in the first trial, we rerun the fit assuming $C_{f}=1$, following the procedure detailed in Misawa et al. (2007a). The fitting results for C IV and N v doublets are shown in Figures 4 and 5, and fitting parameters are summarized in Table 2.

\subsubsection{Image $A$}

In our high-resolution spectrum, the associated C IV absorption line is deblended into at least 16 narrow components at $v_{\mathrm{ej}} \sim-1600 \mathrm{~km} \mathrm{~s}^{-1}-94 \mathrm{~km} \mathrm{~s}^{-1}$ from the quasar emission redshift at $z_{\mathrm{em}} \sim 2.197$, as shown in the $\mathrm{C}$ IV $\lambda 1548$ panel of Figures 3 and 4 . Among the $16 \mathrm{C}$ IV components, all have corresponding Ly $\alpha$ absorption, while only components at $v_{\mathrm{ej}} \geqslant-846 \mathrm{~km} \mathrm{~s}^{-1}$ have corresponding $\mathrm{N} \mathrm{v}$ doublets. Those at 
Table 2

Fitting Parameters of C IV Absorbers

\begin{tabular}{|c|c|c|c|c|c|c|c|c|c|}
\hline (1) & $\begin{array}{l}z_{\mathrm{abs}}{ }^{\mathrm{a}} \\
(2)\end{array}$ & $\begin{array}{c}v_{\mathrm{ej}}^{\mathrm{b}} \\
\left(\mathrm{km} \mathrm{s}^{-1}\right) \\
(3)\end{array}$ & $\begin{array}{c}\log N \\
\left(\mathrm{~cm}^{-2}\right) \\
(4)\end{array}$ & $\begin{array}{c}\sigma(\log N) \\
\left(\mathrm{cm}^{-2}\right) \\
(5)\end{array}$ & $\begin{array}{c}b \\
\left(\mathrm{~km} \mathrm{~s}^{-1}\right) \\
(6)\end{array}$ & $\begin{array}{c}\sigma(b) \\
\left(\mathrm{km} \mathrm{s}^{-1}\right) \\
(7)\end{array}$ & $\begin{array}{l}C_{f}^{\mathrm{c}} \\
(8)\end{array}$ & $\begin{array}{c}+\sigma\left(C_{f}\right) \\
(9)\end{array}$ & $\begin{array}{c}-\sigma\left(C_{f}\right) \\
(10)\end{array}$ \\
\hline \multicolumn{10}{|c|}{ Image A } \\
\hline \multicolumn{10}{|c|}{ CiV } \\
\hline 1 & 2.1800 & -1599.5 & 13.52 & 0.54 & 6.30 & 2.45 & 0.20 & 0.13 & -0.13 \\
\hline 2 & 2.1810 & -1505.2 & 13.80 & 0.29 & 25.52 & 3.01 & 0.38 & 0.25 & -0.18 \\
\hline 3 & 2.1818 & -1429.7 & 14.08 & 0.06 & 18.51 & 1.19 & 0.82 & 0.05 & -0.05 \\
\hline 4 & 2.1822 & -1392.0 & 13.85 & 0.06 & 13.16 & 0.83 & 0.96 & 0.08 & -0.08 \\
\hline 5 & 2.1829 & -1326.0 & 12.97 & 0.98 & 14.35 & 37.5 & $1.00^{\mathrm{d}}$ & $\ldots$ & $\ldots$ \\
\hline 6 & 2.1880 & -845.7 & 13.39 & 0.75 & 16.40 & 3.62 & 0.62 & 2.88 & -0.88 \\
\hline 7 & 2.1886 & -789.3 & 14.28 & 0.15 & 16.08 & 2.18 & 0.49 & 0.05 & -0.05 \\
\hline 8 & 2.1894 & -714.0 & 15.03 & 0.34 & 25.44 & 6.00 & 0.62 & 0.04 & -0.04 \\
\hline 9 & 2.1901 & -648.2 & 14.28 & 0.12 & 19.18 & 2.61 & 0.88 & 0.06 & -0.06 \\
\hline 10 & 2.1910 & -563.6 & 14.28 & 0.09 & 44.19 & 9.87 & $1.00^{\mathrm{d}}$ & $\ldots$ & $\ldots$ \\
\hline 11 & 2.1942 & -262.9 & 14.01 & 0.29 & 143.71 & 113.3 & $1.00^{\mathrm{d}}$ & $\ldots$ & $\ldots$ \\
\hline 12 & 2.1944 & -244.1 & 14.82 & 0.10 & 21.34 & 1.63 & 0.89 & 0.04 & -0.04 \\
\hline 13 & 2.1953 & -159.6 & 14.84 & 0.09 & 34.58 & 5.17 & 0.85 & 0.06 & -0.06 \\
\hline 14 & 2.1964 & -56.3 & 15.65 & 0.20 & 32.62 & 2.83 & 0.82 & 0.04 & -0.04 \\
\hline 15 & 2.1975 & 46.9 & 14.37 & 0.12 & 16.02 & 2.14 & 0.46 & 0.06 & -0.06 \\
\hline 16 & 2.1980 & 93.8 & 14.46 & 0.06 & 34.85 & 2.60 & 0.48 & 0.04 & -0.04 \\
\hline \multicolumn{10}{|l|}{$\mathrm{Nv}$} \\
\hline 1 & 2.1889 & -761.1 & 14.18 & 0.60 & 77.80 & 73.1 & $1.00^{\mathrm{d}}$ & $\ldots$ & $\ldots$ \\
\hline 2 & 2.1894 & -714.0 & 13.94 & 0.85 & 27.88 & 31.5 & $1.00^{\mathrm{d}}$ & $\ldots$ & $\ldots$ \\
\hline 3 & 2.1901 & -648.2 & 14.52 & 0.06 & 31.83 & 3.25 & 0.92 & 0.07 & -0.07 \\
\hline 4 & 2.1908 & -582.4 & 14.99 & 0.39 & 14.50 & 3.05 & 0.88 & 0.07 & -0.07 \\
\hline 5 & 2.1914 & -526.0 & 14.13 & 0.16 & 26.67 & 10.92 & $1.00^{\mathrm{d}}$ & $\ldots$ & $\ldots$ \\
\hline 6 & 2.1938 & -300.4 & 15.25 & 4.07 & 12.93 & 23.71 & 0.11 & 0.07 & -0.07 \\
\hline 7 & 2.1943 & -253.5 & 15.03 & 0.20 & 18.61 & 2.16 & 0.96 & 0.06 & -0.06 \\
\hline 8 & 2.1952 & -169.0 & 14.92 & 0.11 & 36.29 & 8.38 & 0.94 & 0.07 & -0.07 \\
\hline 9 & 2.1962 & -75.1 & 15.11 & 0.15 & 39.00 & 15.02 & 0.90 & 0.06 & -0.06 \\
\hline 10 & 2.1969 & -9.4 & 14.41 & 0.07 & 12.90 & 1.91 & $1.00^{\mathrm{d}}$ & $\ldots$ & $\ldots$ \\
\hline 11 & 2.1978 & 75.1 & 14.69 & 0.08 & 46.82 & 3.58 & 0.61 & 0.07 & -0.07 \\
\hline \multicolumn{10}{|c|}{ Image B } \\
\hline \multicolumn{10}{|c|}{ CiV } \\
\hline 1 & 2.1814 & -1467.4 & 13.37 & 0.41 & 16.46 & 18.15 & $1.00^{\mathrm{d}}$ & $\ldots$ & $\ldots$ \\
\hline 2 & 2.1821 & -1401.4 & 14.20 & 0.04 & 13.75 & 0.37 & 0.99 & 0.04 & -0.04 \\
\hline 3 & 2.1827 & -1344.9 & 13.81 & 0.32 & 21.73 & 3.05 & 0.31 & 0.17 & -0.15 \\
\hline 4 & 2.1886 & -789.3 & 15.96 & 1.03 & 17.73 & 4.40 & 0.26 & 0.05 & -0.05 \\
\hline 5 & 2.1896 & -695.2 & 14.45 & 0.17 & 38.57 & 5.69 & 0.76 & 0.05 & -0.05 \\
\hline 6 & 2.1901 & -648.2 & 14.18 & 0.14 & 18.49 & 2.90 & 0.77 & 0.14 & -0.14 \\
\hline 7 & 2.1910 & -563.6 & 14.09 & 0.18 & 37.21 & 11.44 & $1.00^{\mathrm{d}}$ & $\ldots$ & $\ldots$ \\
\hline 8 & 2.1944 & -244.1 & 14.67 & 0.07 & 22.64 & 1.35 & 0.85 & 0.04 & -0.04 \\
\hline 9 & 2.1952 & -169.0 & 15.51 & 3.21 & 6.63 & 5.29 & 0.74 & 0.06 & -0.06 \\
\hline 10 & 2.1959 & -103.2 & 15.03 & 0.06 & 81.86 & 6.01 & 0.77 & 0.06 & -0.06 \\
\hline 11 & 2.1970 & 0.0 & 14.29 & 0.15 & 26.03 & 4.26 & 0.54 & 0.14 & -0.14 \\
\hline 12 & 2.1971 & 9.4 & 15.52 & 0.23 & 67.71 & 6.19 & 0.27 & 0.04 & -0.04 \\
\hline \multicolumn{10}{|l|}{$\mathrm{Nv}$} \\
\hline 1 & 2.1884 & -808.1 & 13.44 & 0.78 & 20.68 & 37.5 & $1.00^{\mathrm{d}}$ & $\ldots$ & $\ldots$ \\
\hline 2 & 2.1897 & -685.8 & 14.89 & 0.04 & 51.32 & 1.50 & 0.84 & 0.05 & -0.05 \\
\hline 3 & 2.1909 & -573.0 & 15.19 & 0.40 & 13.57 & 2.11 & 0.90 & 0.05 & -0.05 \\
\hline 4 & 2.1915 & -516.6 & 13.95 & 0.20 & 19.20 & 10.0 & $1.00^{\mathrm{d}}$ & $\ldots$ & $\ldots$ \\
\hline 5 & 2.1943 & -253.5 & 14.58 & 0.13 & 20.22 & 1.92 & 0.99 & 0.06 & -0.06 \\
\hline 6 & 2.1946 & -225.3 & 15.30 & 1.85 & 9.69 & 7.26 & 0.86 & 0.07 & -0.07 \\
\hline 7 & 2.1952 & -169.0 & 14.65 & 0.07 & 24.92 & 5.53 & 0.98 & 0.06 & -0.06 \\
\hline 8 & 2.1957 & -122.0 & 14.54 & 0.17 & 14.51 & 4.85 & 0.93 & 0.08 & -0.08 \\
\hline 9 & 2.1965 & -46.9 & 15.34 & 0.29 & 34.20 & 8.34 & 0.91 & 0.05 & -0.05 \\
\hline 10 & 2.1972 & 18.8 & 14.92 & 0.35 & 20.74 & 3.18 & 0.65 & 0.08 & -0.08 \\
\hline 11 & 2.1980 & 93.8 & 14.87 & 0.35 & 15.78 & 3.11 & 0.35 & 0.05 & -0.05 \\
\hline
\end{tabular}

Notes.

${ }^{a}$ Absorption redshift.

${ }^{\mathrm{b}}$ Ejection velocity from the quasar emission redshift, with negative values denoting blueshifted line.

${ }^{\mathrm{c}}$ Covering factor.

${ }^{\mathrm{d}}$ Fitting the component, assuming $C_{f}=1$. 

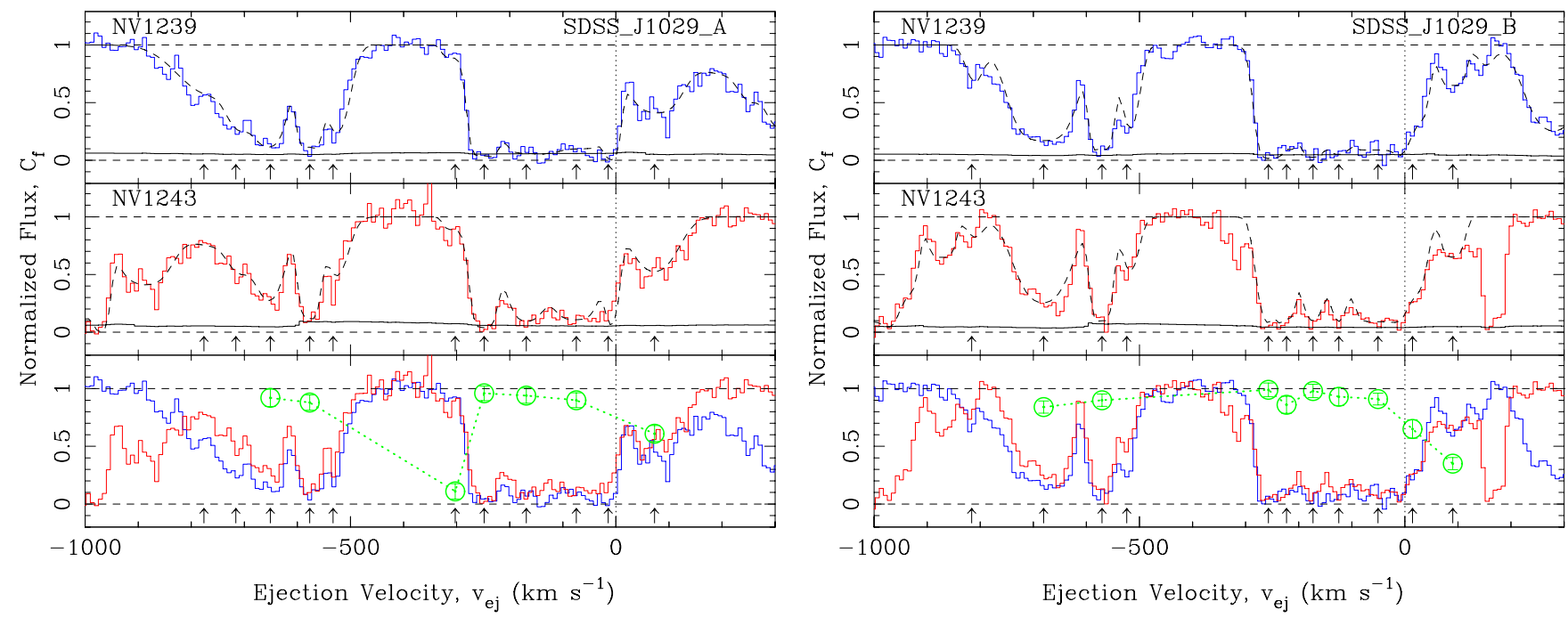

Figure 5. Same as Figure 4, but for N v doublets.

(A color version of this figure is available in the online journal.)

$v_{\text {ej }} \sim-1400 \mathrm{~km} \mathrm{~s}^{-1}$ have only subtle $\mathrm{N} \mathrm{v}$ doublets. It is difficult to tell whether or not $\mathrm{Si}$ IV doublets corresponding to the C IV system exist because the Si IV $\lambda 1394$ blends with Si II $\lambda 1527$ at $z_{\text {abs }}=1.9115$ and 1.9141, and because the Si IV $\lambda 1403$ is also severely contaminated by $\mathrm{C}$ IV doublets at $z_{\mathrm{abs}}=1.8909-1.9141$.

The absorption component at $v_{\mathrm{ej}} \sim 800 \mathrm{~km} \mathrm{~s}^{-1}$ in the C IV $\lambda 1548$ panel is not $\mathrm{C}$ IV but instead Fe II $\lambda 2344$ at $z_{\text {abs }} \sim 0.9187$. We also confirm that a strong absorption feature at $\lambda \sim 4900 \AA$ only seen in image A of Figure 2 is Fe II $\lambda 2600$ at the same redshift. It follows that the differential absorption profile of the associated $\mathrm{C}$ IV absorption line between images $\mathrm{A}$ and $\mathrm{B}$ seen in low-resolution spectra (Figure 2) is mainly due to these physically unrelated Fe II lines.

\subsubsection{Image $B$}

The associated $\mathrm{C}$ IV line is deblended into 12 narrow components at $v_{\mathrm{ej}} \sim-1467-9.4 \mathrm{~km} \mathrm{~s}^{-1}$ from the quasar emission redshift as shown in the C IV $\lambda 1548$ panel of Figures 3 and 4. Their general profile is similar to that of the image A. Among the 12 components, all have corresponding Ly $\alpha$, while only those at $v_{\mathrm{ej}}>-789 \mathrm{~km} \mathrm{~s}^{-1}$ have corresponding $\mathrm{N} v$ doublets. On the other hand, only two C IV components at $v_{\mathrm{ej}} \sim-1467 \mathrm{~km} \mathrm{~s}^{-1}$ and $-1401 \mathrm{~km} \mathrm{~s}^{-1}$ have Si IV doublets. The ionization condition of the components at $v_{\mathrm{ej}}>$ $-789 \mathrm{~km} \mathrm{~s}^{-1}$ is clearly higher than those at $v_{\mathrm{ej}} \sim-1467 \mathrm{~km} \mathrm{~s}^{-1}$ and $-1401 \mathrm{~km} \mathrm{~s}^{-1}$.

The Si IV $\lambda 1403$ lines blend with a clustering of C IV lines at $z_{\text {abs }} \sim 1.90$. Absorption features at $v_{\mathrm{ej}} \sim-700 \mathrm{~km} \mathrm{~s}^{-1}$ in the Si IV $\lambda 1394$ panel and at $v_{\text {ej }} \sim 200 \mathrm{~km} \mathrm{~s}^{-1}$ in the $\mathrm{N} v \lambda 1243$ panel are Si II $\lambda 1527$ at $z_{\text {abs }}=1.9118$ and $\mathrm{C}$ II $\lambda 1335$ at $z_{\mathrm{abs}}=$ 1.9788 , respectively.

\subsubsection{Other Lines}

Because our spectra cover a wide range of wavelength, we also identify all $\mathrm{C}$ IV, $\mathrm{Si}$ IV, and $\mathrm{N} v$ doublets from $3800 \AA-4210 \AA$ on blue CCD chip and from $4280 \AA-5110 \AA$ on red CCD chip, avoiding some unusable regions (4155 $\mathrm{A}-4160 \AA$ and $4620 \AA-4740 \AA$ ), Ly $\alpha$ forest, and bad CCD columns. Toward image A, we identify $11 \mathrm{C}$ IV doublets at $z_{\mathrm{abs}}=1.6151$, $1.8909,1.8956,1.8983,1.9115,1.9133,1.9141,1.9322,2.1084$, $2.1270,2.1350$ and $2 \mathrm{Si}$ IV doublets at $z_{\mathrm{abs}}=1.9115$ and
1.9138 with $\geqslant 5 \sigma$ detection in addition to the associated system. We also find one Fe II system identified with Fe II $\lambda 2344$, Fe II $\lambda 2374, \mathrm{Fe}$ II $\lambda 2383, \mathrm{Fe}$ II $\lambda 2587, \mathrm{Fe}$ II $\lambda 2600$ at $z_{\mathrm{abs}}$ $\sim 0.9187$ as described above. Toward image B, we identified $15 \mathrm{C}$ IV doublets at $z_{\text {abs }}=1.6148,1.6556,1.6925,1.7065$, $1.8910,1.8950,1.8972,1.9018,1.9118,1.9322,1.9788,2.1078$, $2.1085,2.1210,2.1284$ and $4 \mathrm{Si}$ IV doublets at $z_{\mathrm{abs}}=1.8941$, $1.8949,1.8975,1.9018$ with $\geqslant 5 \sigma$ detection in addition to the associated system.

\section{DISCUSSION}

In this section, we discuss the origin (i.e., intrinsic or intervening) of the associated absorption lines (Section 4.1), possible geometries toward images A and B (Section 4.2), and models of single/multiple sight line(s) (Section 4.3), respectively.

\subsection{Intrinsic or Intervening}

While BALs have a high probability of being physically associated to the quasars, NALs are difficult to classify as intrinsic or intervening. With our high-resolution spectra, the associated C IV absorption line is deblended into multiple narrow components. As for $\mathrm{C}$ IV and $\mathrm{N} v$ doublets (whose absorption features are clearly detected without being affected by any data defects or line blending), we measure their physical parameters by performing Voigt profile fitting with MINFIT. We have several observational results that support an intrinsic origin for these features, itemized below in order of relevance.

(1) The associated C IV NALs show clear evidence of partial coverage (see Figure 4 and Table 2). Covering factors are quite important for ascertaining the location of the absorbers. Among 16 and $12 \mathrm{C}$ IV components in the spectra of images A and B, 6 and 6 components show partial coverage at the $4 \sigma$ level (i.e., $C_{f}+4 \sigma\left(C_{f}\right) \lesssim$ 1.0), respectively. This supports the physical sizes of the absorbers being comparable to or even smaller than the size of the background source, suggesting that the system is intrinsic to the quasar itself (e.g., Hamann et al. 1997a; Barlow \& Sargent 1997; Crenshaw et al. 1999). Interestingly, $\mathrm{N} v$ NALs have higher $C_{f}$ values and are consistent with full coverage with only a few exceptions. 
Covering factors are not necessarily identical for all ions from the same absorber, both in BALs (e.g., Hamann et al. 1997b) and NALs (e.g., Misawa et al. 2007a). In the literature, ions in higher ionization states usually have larger $C_{f}$, as is also the case for the associated system in SDSS J1029+2623.

(2) The associated C IV NALs show the so-called line-locking phenomenon (i.e., blue components of C IV doublets are aligned with red ones of the following doublets; see Figures 3 and 4). Chance coincidence of such alignments is negligibly small. This is naturally explained by radiative acceleration (e.g., Perry et al. 1978; Weymann et al. 1981; Foltz et al. 1987). Although several mechanisms have been proposed to explain the acceleration of outflowing winds, radiative acceleration almost always contributes substantially. Line locking requires that the sight lines be approximately parallel to the gas motion.

(3) The velocity distribution of the associated $\mathrm{C}$ IV and $\mathrm{N} \mathrm{V}$ NALs shows values beyond $1000 \mathrm{~km} \mathrm{~s}^{-1}$, which is too large for an origin in IGM, foreground galaxy, and ISM of the host galaxy. Intervening absorption lines are typically clustered in $\Delta v<400 \mathrm{~km} \mathrm{~s}^{-1}$ both for metal lines (e.g., Sargent et al. 1980; Young et al. 1982; Petitjean \& Bergeron 1994; Churchill \& Vogt 2001; Pichon et al. 2003) and for H I lines (e.g., Lu et al. 1996; Misawa et al. 2004; Penton et al. 2004). The ISM of most galaxies show velocities below this limit. A distribution with velocities $\geqslant 1000 \mathrm{~km} \mathrm{~s}^{-1}$ strongly supports an intrinsic origin due to the outflow wind.

(4) These NALs have small ejection velocities from the quasar emission redshift, $\left|v_{\mathrm{ej}}\right| \leqslant 1600 \mathrm{~km} \mathrm{~s}^{-1}$. Wise et al. (2004) found that a high fraction $(\sim 21 \%)^{15}$ of the associated NALs within $v_{\mathrm{ej}}=5000 \mathrm{~km} \mathrm{~s}^{-1}$ from the quasars is time variable (i.e., intrinsic lines). Nestor et al. (2008) discovered an excess number density of C IV NALs in quasar vicinities, and concluded that almost half of absorbers at $\left|v_{\mathrm{ej}}\right| \leqslant$ $12,000 \mathrm{~km} \mathrm{~s}^{-1}$ are intrinsic to the quasars, with a peak value of $\sim 80 \%$ at $\left|v_{\mathrm{ej}}\right| \sim 2000 \mathrm{~km} \mathrm{~s}^{-1}$.

\subsection{Possible Geometries}

Based on multiple medium-resolution spectra, we confirmed that the associated $\mathrm{C}$ IV absorption lines toward both images $A$ and $B$ retain their profiles unchanged, while their profiles are clearly different each other. This result was originally expected to reject the time-variation scenario (i.e., scenario I). However, with the high-resolution spectrograph, the associated C IV absorption lines are deblended into multiple narrow components, of which two are foreground absorption lines of Fe II $\lambda 2344$ and Fe II $\lambda 2600$ at $z_{\text {abs }} \sim 0.9187$. We confirmed that the differential $\mathrm{C}$ IV absorption profiles between the lensed images seen in medium-resolution spectra are mainly due to these physically unrelated lines. Thus, we cannot distinguish different scenarios with medium-resolution spectra alone.

High-resolution spectra taken with Subaru/HDS provide us with several important clues regarding the origin of the associated C IV lines. First of all, the general profiles of the C IV absorption lines toward images $\mathrm{A}$ and $\mathrm{B}$ are very similar to each other. This means that the size of the absorber must be larger than the transverse distance of the two sight lines. In the extreme case, the absorber's location would be very close to the background flux source so as to make the separation angle almost negligible.

\footnotetext{
15 This is a lower limit on the fraction since not all systems necessarily show time variation.
}

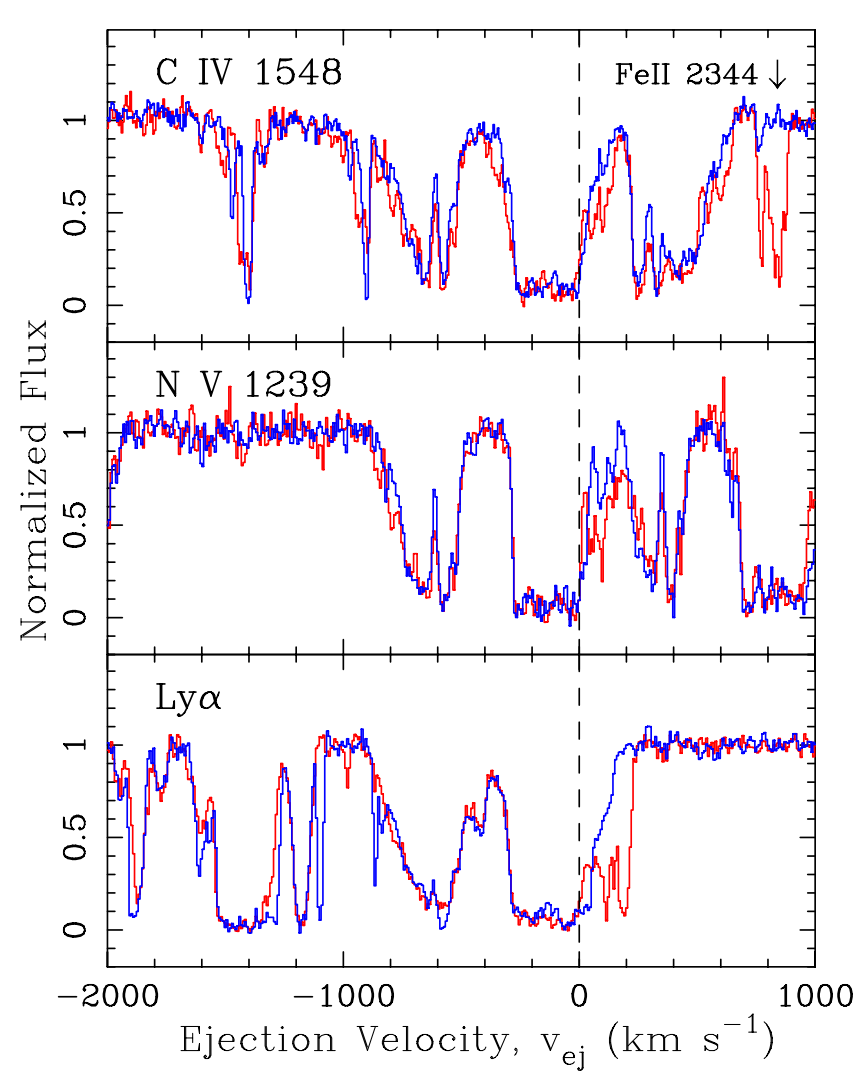

Figure 6. Comparison of the associated $\mathrm{C}$ IV, $\mathrm{N} \mathrm{v}$, and Ly $\alpha$ absorption profiles toward image $\mathrm{A}$ (red) and image B (blue).

(A color version of this figure is available in the online journal.)

Second, we see a clear difference of absorption profiles between images $\mathrm{A}$ and $\mathrm{B}$ in various structures. For example, at $v_{\mathrm{ej}} \sim$ $0-200 \mathrm{~km} \mathrm{~s}^{-1}$, there exist additional absorption components in C IV, N v, Ly $\alpha$ panels of Figure 6 only in image A. This small difference, discovered only after taking high-resolution spectra, reminds us of the original question: What is the source of this difference? Finally, the most important clue is that a substantial fraction of absorption components show partial coverage, which means that the physical sizes of the absorbers are smaller than or comparable to the background source such as the continuum source and the BELR. While some intrinsic absorption lines cover only the continuum source (e.g., Arav et al. 1999), the covering factors toward the continuum source and the BELR usually take specific values, depending on the relative strengths of their fluxes (Ganguly et al. 1999). In our case, the residual flux at $\lambda \sim 4948 \AA$, at which the C IV emission line peaks, is almost zero. This means that absorbers as a whole cover both the continuum source and the BELR significantly. Therefore, size estimation of the flux sources is important.

We estimate the size of the BELR $\left(R_{\mathrm{BELR}}\right)$ using the empirical relation between $R_{\mathrm{BELR}}$ and quasar luminosity. This relation was originally discovered through reverberation mapping (Kaspi et al. 2000) and then extended to brighter quasars with monochromatic luminosities of $>10^{44} \mathrm{ergs} \mathrm{s}^{-1}$ to redshifts $z_{\mathrm{em}}>0.7$ (Equation (A4) in McLure \& Dunlop 2004). The monochromatic luminosity of image $\mathrm{A}$ at $\lambda_{r}=3000 \AA$ in the quasar rest frame is measured in Shen et al. (2011) as $\log \lambda L_{3000}=46.21 \mathrm{erg} \mathrm{s}^{-1}$. After correcting the magnification factor of image $\mathrm{A}, \mu_{A}=10.4$ (Oguri et al. 2012), we estimate $R_{\mathrm{BELR}}$ to be $\sim 0.09_{-0.05}^{+0.09} \mathrm{pc}$, where the main source of $1 \sigma$ 

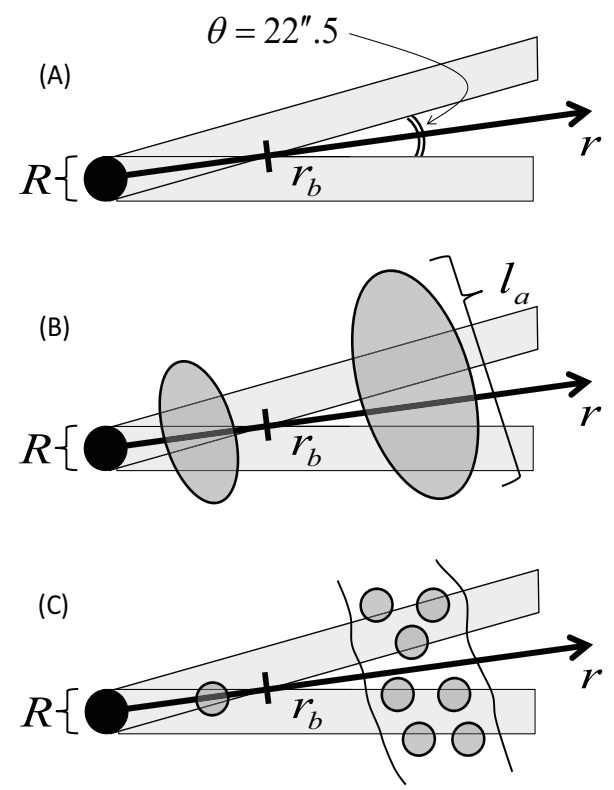

Figure 7. Possible geometries that could lead to the small differential profiles of the associated absorption lines toward the two lensed images of SDSS $\mathrm{J} 1029+2623$. General geometry of two sight lines (A), geometrical models for a single sight line (B), and multiple sight lines (C) are shown from top to bottom. A filled black circle with a size of $R$ and meshed ovals/circles with a size of $l_{a}$ denote the central flux source and absorbers. The radial distance $r_{b}$ is the boundary distance. A region surrounded by two curves in (C) is a filamentary/sheet-like structure.

uncertainty comes from the scatter in the empirical relation in McLure \& Dunlop (2004).

As to the size of the continuum source $\left(R_{\text {cont }}\right)$, we take five times the Schwarzschild radius, $5 R_{S}=10 G M_{\mathrm{BH}} / c^{2}$, following Misawa et al. (2005), where $R_{S}$ and $M_{\mathrm{BH}}$ are the Schwarzschild radius and mass of the central $\mathrm{BH}$. The virial mass of the central $\mathrm{BH}$ is already calculated in Shen et al. (2011) using the luminosity and FWHM of broad emission lines of image A. Again, after correcting for magnification, we obtain $\log M_{\mathrm{BH}} / M_{\odot}=8.72$, and then $R_{\text {cont }}$ is evaluated to be $\sim 2.54 \times 10^{-4} \mathrm{pc}$. This is almost 300 times smaller than $R_{\mathrm{BELR}}$.

The size of the background flux source $(R)$ and the absorber's distance from the central flux source $(r)$ decide geometry, i.e., a single sight line or multiple sight lines. The transverse distance of the two sight lines ( $a$ ) can be calculated as $a=r \theta$, where $\theta$ is the separation angle of the two sight lines seen from the flux source (which is very similar to the separation angle seen from us, $\left.\theta \sim 22^{\prime \prime} .5\right)$. At a distance, where $R \leqslant a(=r \theta)$ is satisfied, the two sight lines become fully separated with no overlap. We call this distance a boundary distance $\left(r_{b}\right)$, hereafter (see Figure $7(\mathrm{~A})$ ). The boundary distance is $r_{b} \sim 2.3 \mathrm{pc}$ if only the continuum source is the flux source, and $\sim 788 \mathrm{pc}$ if the BELR is also the background source. The latter is comparable to the distance of outflowing gas measured by de Kool et al. (2001) and Hamann et al. (2001) for other quasars. The geometry also depends on the absorber's size $\left(l_{a}\right)$. If the size of the absorber (whose internal sub-structure is ignored here) is much larger than the transverse distance (i.e., $l_{a} \gg r \theta$ ), a single sight line scenario is applicable regardless of the absorber's distance from the flux source (see Figure 7(B)). However, this geometry cannot be applied to our case because the partial coverage we found in Section 3.2 requires the size of each absorber to be comparable or smaller than the size of background source. On the other hand, if $l_{a} \leqslant r \theta$ (Figure 7(C)), we should analyze the condition in more detail. In the case of $l_{a} \leqslant r \theta \ll R$ (i.e., the absorber's distance is smaller than the boundary distance), a substantial fraction of both sight lines is covered by a single absorber although the fraction depends on the distance and the absorber's size. Small differences of absorption profiles, seen in the high-resolution spectra of images A and B, can be explained either by (1) a different part of the absorber's outskirts covering each sight line (quasi-scenario II) or by (2) time variation (scenario I). In the case of $l_{a} \leqslant R \leqslant r \theta$ (i.e., the absorber's distance is larger than the boundary distance), this will be bona-fide scenario II. One shortcoming of this model is that we cannot reproduce common absorption profiles seen in the two sight lines because the same absorber cannot cover both sight lines. We will discuss this problem later.

\subsection{Single Sight Line or Multiple Sight Lines}

Hamann et al. (2011) claimed that NAL absorbers should be located in a low gravity environment far from the central $\mathrm{BH}$ in order to maintain kinematic stability. Their result suggests that the ejection velocity of NAL absorbers is larger than the escape velocity so that the absorber is gravitationally unbound. If the NAL absorbers in our target quasar are also in a similar kinematic condition, their radial distance from the central $\mathrm{BH}$ should be $r \geqslant 1.79 \mathrm{pc}$. This is close to the boundary distance in the case that only the continuum source is the background flux source. As a result, we do not have any conclusive evidence to either accept or reject scenarios I or II. Therefore, we will discuss the absorber's physical condition further assuming both scenarios in turn below.

\subsubsection{Single Sight Line (Scenario I)}

If scenario I represents reality, there are two possible origins of time variability, (1) a change of ionization condition of the absorber (e.g., Hamann et al. 2011; Misawa et al. 2007b) and (2) the absorber moving across our sight lines to change the covering factor (e.g., Hamann et al. 2008; Gibson et al. 2008). Neither situation is applicable for the case of intervening absorbers as discussed in Narayanan et al. (2004).

If a change in ionization is the origin of variation, we can place constraints on the electron density and the distance from the flux source following the procedure by Hamann et al. (1997a) and Narayanan et al. (2004). We cannot evaluate the specific ionization condition because a wide range of ionic species (which is necessary for photoionization modeling) are not detected in our spectra. Therefore, by adopting the following assumptions: (1) the gas is very close to ionization equilibrium, (2) the change of ionizing flux is small, and (3) $\mathrm{C}$ IV is the dominant ionization stage of carbon, we estimate the electron density to be $n_{e} \sim 1 / \alpha \Delta t \sim 1.78 \times 10^{4} \mathrm{~cm}^{-3}$ by assuming a variation timescale in the quasar rest frame $(\sim 233$ days) as a recombination time $(\Delta t)$, where we use the recombination coefficient of $\mathrm{C}$ IV $\rightarrow \mathrm{C}$ III in gas temperature of 20,000 K (Hamann et al. 1995). Because the variation timescale is an upper limit of the recombination time, we should regard the electron density as a lower limit. We can also evaluate the distance of the absorber from the flux source using the prescription of Narayanan et al. (2004). The ionization parameter $(U)^{16}$ depends on the bolometric luminosity of the quasar, the continuum shape, distance from the flux source, and

\footnotetext{
16 The ionization parameter $U$ is defined as the ratio of hydrogen ionizing
} photon density $\left(n_{\gamma}\right)$ to the electron density $\left(n_{e}\right)$, i.e., $U \sim n_{\gamma} / n_{e}$. 
electron density. By adopting the continuum shape of Narayanan et al. (2004), we estimate the distance of the absorber to be $r \leqslant 0.44 \mathrm{kpc}$, with an ionization parameter of $U=0.02$ (in which $\mathrm{C}$ IV is the dominant ionization stage; Hamann et al. $1995,1997 b)$, and the bolometric luminosity of the quasar of $\log L_{\text {bol }}=45.87 \mathrm{erg} \mathrm{s}^{-1}$ (Shen et al. 2011). The limits on the electron density and the absorber's distance are consistent with the literature (e.g., Narayanan et al. 2004; Misawa et al. 2005).

If the gas motion (i.e., crossing of our sight line) is the origin of variation, we can simply estimate the average crossing velocity $\left(v_{\text {cross }} \sim R / \Delta t\right)$ to be $\sim 390 \mathrm{~km} \mathrm{~s}^{-1}$ if the continuum source is the only background source. Again, this is the minimum velocity because $\Delta t$ is an upper limit on the variability timescale. The BELR should not be the flux source, because the corresponding crossing velocity, $\sim 1.32 \times 10^{5} \mathrm{~km} \mathrm{~s}^{-1}$, is comparable to the speed of light. Thus, we conclude that the absorber covers primarily the UV continuum source with only a small part of the BELR if the gas motion is the origin of variation.

\subsubsection{Multiple Sight Lines (Scenario II)}

The main problem of the bona fide scenario II model, in which the absorber's size is smaller than the transverse distance of the two sight lines, is that we cannot reproduce closely similar absorption profiles seen in the two sight lines because the same absorbers cannot cover both sight lines. A possible solution for this is that there exists a number of clumpy absorbers or fluctuations in gas density (whose sizes are smaller than the background source) constituting a filamentary (or a sheet-like) structure that covers both sight lines (Figure $7(\mathrm{C})$ ). Such a structure is well reproduced above the main body of the outflow by hydrodynamical simulations (e.g., Ohsuga et al. 2005; Proga et al. 1998, 1999).

Here, we calculate the radial mass-outflow rate $(\dot{M})$ toward the two sight lines summing contributions from all absorption components as

$$
\dot{M}=\sum_{i}^{n} C_{f}(i) v_{\mathrm{ej}}(i) R\left(\frac{N_{\mathrm{H}}(i)}{\Delta r(i)}\right) m_{p}
$$

where $N_{\mathrm{H}}(i)$ and $\Delta r(i)$ are the total $\left(\mathrm{H}_{\mathrm{I}}+\mathrm{H}\right.$ II) hydrogen column density and the radial depth of the $i$ th absorption component toward a sight line. $R$ and $m_{p}$ are the size of background source and the proton mass. In Section 3.2, we measured ejection velocities of all $\mathrm{C}$ IV and $\mathrm{N} v$ absorption components by applying Voigt profile fitting. However, their absolute values could be underestimated because emission redshifts determined from broad UV emission lines are systematically blueshifted from the systemic redshift, as measured by narrow, forbidden lines (see, e.g., Corbin 1990; Tytler \& Fan 1992; Brotherton et al. 1994; Marziani et al. 1996). Tytler \& Fan (1992) find a mean blueshift of the broad UV emission lines relative to the systemic redshift of about $260 \mathrm{~km} \mathrm{~s}^{-1}$ and that $90 \%$ of the blueshifts are between 0 and $650 \mathrm{~km} \mathrm{~s}^{-1}$. We therefore add $260 \mathrm{~km} \mathrm{~s}^{-1}$ to the $v_{\text {ej }}$ values given in Table 2 for the calculation herein.

Because our spectra do not detect a wide range of ionic species (which are necessary for photoionization modeling, as described in Section 4.3.1), we can measure neither the ionization condition nor the total hydrogen column density $N(\mathrm{H})$. These parameters decide an absorber's metallicity and radial depth. Therefore, we simply calculate $\dot{M}$ assuming all absorption components have the same ionization condition, metallicity, and radial depth toward both the sight lines as follows:

$$
\begin{aligned}
\dot{M} & =\sum_{i}^{n} C_{f}(i) v_{\mathrm{ej}}(i) R\left(\frac{N_{\mathrm{ion}}(i) \times \mathrm{MIC}_{\mathrm{ion}}}{\Delta r(i)}\right) m_{p} \\
& \propto \sum_{i}^{n} C_{f}(i) v_{\mathrm{ej}}(i) N_{\mathrm{ion}}(i),
\end{aligned}
$$

where MIC (a metallicity-ionization correction) for C IV and $\mathrm{N} v$ are defined as follows, respectively,

$$
\begin{aligned}
\mathrm{MIC}_{\mathrm{C} \text { IV }} & =\frac{N(\mathrm{H})}{N(\mathrm{C})} \times \frac{N(\mathrm{C})}{N(\mathrm{C} \text { IV })}=\frac{N(\mathrm{H})}{N(\mathrm{C} \text { IV })}, \\
\mathrm{MIC}_{\mathrm{Nv}} & =\frac{N(\mathrm{H})}{N(\mathrm{~N})} \times \frac{N(\mathrm{~N})}{N(\mathrm{~N} \mathrm{v})}=\frac{N(\mathrm{H})}{N(\mathrm{~N} \mathrm{v})} .
\end{aligned}
$$

Based on the $\mathrm{C}$ IV and $\mathrm{N} v$ absorption lines, a difference of radial mass-outflow rates toward images $\mathrm{A}$ and $\mathrm{B}$ is only $\sim 30 \%-35 \%$, which suggests that internal fluctuation of the NAL absorber is almost negligible within the angular distance of $\theta \sim 22$.'5. Because $\sim 20 \%-50 \%$ of quasars have at least one intrinsic NAL absorber in their spectra (Vestergaard 2003; Wise et al. 2004; Misawa et al. 2007a; Nestor et al. 2008), the global covering factor of the central flux source surrounded by NAL absorbers can have values of $\Omega \leqslant 0.8 \pi-2 \pi$ in terms of solid angle. Thus, small areas within $\sim 0.002 \%-0.005 \%$ of the total solid angle for NAL absorbers produce similar absorption profiles with the same order of total column densities. In other words, the outflow wind corresponding to the NAL absorbers can be divided into $\leqslant 20,000-50,000$ small zones with common physical conditions.

\section{SUMMARY AND FUTURE WORK}

We carried out medium-resolution spectroscopy of the two brightest images of the quasar SDSS J1029+2623 to see whether or not the associated C IV absorption lines are variable, and also high-resolution spectroscopy in order to measure line parameters and place strict constraints on the absorber's physical conditions. Our main results are as follows.

1. The associated C IV absorption profile does not show clear time variation toward either sight lines in mediumresolution spectra. With high-resolution spectra, we confirmed that the differential line profile is mainly due to the effect of Fe II $\lambda 2344$ and Fe II $\lambda 2600$ lines which arise in a physically unrelated system at $z_{\text {abs }} \sim 0.9187$.

2. In high-resolution spectra, the associated C IV absorption line is deblended into more than 10 narrow components, showing (1) partial coverage, (2) line-locking, (3) large velocity distribution, and (4) small ejection velocity. All of these results support the associated absorption line being physically related to the quasar (i.e., intrinsic absorption line).

3. The associated absorber probably covers both the continuum source and the BELR because the residual flux at $\lambda \sim 4948 \AA$, at which the C IV emission line peaks, is almost zero. The size of the continuum source and the BELR are evaluated to be $R_{\text {cont }} \sim 2.54 \times 10^{-4} \mathrm{pc}$ and $R_{\mathrm{BELR}} \sim 0.09_{-0.05}^{+0.09} \mathrm{pc}$, respectively. If the associated absorber covers both the flux sources toward our sight lines, their typical scale should be comparable to or smaller than $R_{\text {BELR }}$ because they show partial coverage. 
4. The geometry of the associated absorber depends on the sizes of the background flux source $(R)$ and the absorber $\left(l_{a}\right)$, and the absorber's distance from the center $(r)$. If a single sight line model represents reality, the differential line profile implies time variation of the absorber. If a change of ionization level is the origin of the variation, we can place constraints on the absorber's electron density, $n_{e} \leqslant 1.78 \times 10^{4} \mathrm{~cm}^{-3}$, and the absorber's distance from the continuum source, $r \leqslant 0.44 \mathrm{kpc}$. If gas motion is the origin of variation, the continuum source is the only background source because the estimated crossing velocity is too large (comparable to the speed of light) if the BELR is also the flux source.

5. If multiple sight line model is the real scenario, the following condition should be satisfied; the absorber's size $\left(l_{a}\right) \leqslant$ the size of the background flux source $(R) \leqslant$ transverse distance of the two sight lines $(a)$. However, such a condition cannot allow a single absorber to cover both sight lines, in order to reproduce similar absorption profiles as seen in the high-resolution spectra. A filamentary (or a sheet-like) structure made of multiple clumpy gas clouds could solve this issue. Indeed, such structures are reproduced by hydrodynamical simulations.

In order to assess the origin of the associated absorber further, we need to obtain high-resolution spectra again. We have shown that monitoring observations with medium-resolution spectra are not useful because the associated $\mathrm{C}$ IV absorption line in the image $\mathrm{A}$ is significantly contaminated with physically unrelated foreground Fe II absorption lines. Additional high-resolution spectra will provide us with stronger evidence for scenario I if we see clear time variations or for scenario II if the images do not show variability.

T.M. acknowledges support from the Special Postdoctoral Research Program of RIKEN. The research was partially supported by the Japan Society for the Promotion of Science through Grant-in-Aid for Scientific Research 23740148 and Shinshu University Research Grant for Exploratory Research by Young Scientists. This work was also supported in part by the FIRST program "Subaru Measurements of Images and Redshifts (SuMIRe)," World Premier International Research Center Initiative (WPI Initiative), MEXT, Japan, and Grantin-Aid for Scientific Research from the JSPS (23740161). We also thank Kentaro Aoki for his comments about data analysis, and Chris Churchill for providing us with MINFIT. We also thank the anonymous referee for helpful comments and suggestions.

\section{REFERENCES}

Arav, N., Becker, R. H., Laurent-Muehleisen, S. A., et al. 1999, ApJ, 524, 566 Balsara, D. S., \& Krolik, J. H. 1993, ApJ, 402, 109

Barlow, T. A., \& Sargent, W. L. W. 1997, AJ, 113, 136

Becker, R. H., Gregg, M. D., Hook, I. M., et al. 1997, ApJL, 479, 93

Becker, R. H., White, R. L., Gregg, M. D., et al. 2001, ApJS, 135, 227

Blandford, R. D., \& Payne, D. G. 1982, MNRAS, 199, 883

Brotherton, M. S., Wills, B. J., Steidel, C. C., \& Sargent, W. L. W. 1994, ApJ, 423, 131

Capellupo, D. M., Hamann, F., Shields, J. C., Rodríguez Hidalgo, P., \& Barlow, T. A. 2012, MNRAS, 422, 3249

Chartas, G. 2000, ApJ, 531, 81

Chelouche, D., \& Netzer, H. 2005, ApJ, 625, 95

Churchill, C. W. 1997, PhD thesis, University of California, Santa Cruz

Churchill, C. W., \& Vogt, S. S. 2001, AJ, 122, 679

Churchill, C. W., Vogt, S. S., \& Charlton, J. C. 2003, AJ, 125, 98
Corbin, M. R. 1990, ApJ, 357, 346

Crenshaw, D. M., Kraemer, S. B., Boggess, A., et al. 1999, ApJ, 516, 750

Crotts, A. P. S., \& Fang, Y. 1998, ApJ, 502, 16

de Kool, M., Arav, N., Becker, R. H., et al. 2001, ApJ, 548, 609

de Kool, M., \& Begelman, M. C. 1995, ApJ, 455, 448

Elvis, M. 2000, ApJ, 545, 63

Emmering, R. T., Blandford, R. D., \& Shlosman, I. 1992, ApJ, 385, 460

Everett, J. E. 2005, ApJ, 631, 689

Fohlmeister, J., Kochanek, C. S., Falco, E. E., et al. 2012, arXiv:1207.5776

Foltz, C. B., Weymann, R. J., Morris, S. L., \& Turnshek, D. A. 1987, ApJ, 317,450

Gabel, J. R., Arav, N., \& Kim, T.-S. 2006, ApJ, 646, 742

Ganguly, R., Eracleous, M., Charlton, J. C., \& Churchill, C. W. 1999, AJ, 117,2594

Gibson, R. R., Brandt, W. N., Schneider, D. P., \& Gallagher, S. C. 2008, ApJ, 675,985

Gibson, R. R., Jiang, L., Brandt, W. N., et al. 2009, ApJ, 692, 758

Green, P. J. 2006, ApJ, 644, 733

Hamann, F., Barlow, T. A., Beaver, E. A., et al. 1995, ApJ, 443, 606

Hamann, F., Barlow, T. A., \& Junkkarinen, V. 1997a, ApJ, 478, 87

Hamann, F., Barlow, T. A., Junkkarinen, V., \& Burbidge, E. M. 1997b, ApJ, 478,80

Hamann, F., Kanekar, N., Prochaska, J. X., et al. 2011, MNRAS, 410, 1957

Hamann, F., Kaplan, K. F., Rodríguez Hidalgo, P., Prochaska, J. X., \& HerbertFort, S. 2008, MNRAS, 391, L39

Hamann, F., \& Sabra, B. 2004, in ASP Conf. Ser. 311, AGN Physics with the Sloan Digital Sky Survey, ed. G. T. Richards \& P. B. Hall (San Francisco, CA: ASP), 203

Hamann, F. W., Barlow, T. A., Chaffee, F. C., Foltz, C. B., \& Weymann, R. J. 2001, ApJ, 550, 142

Hewett, P. C., \& Foltz, C. B. 2003, AJ, 125, 1784

Inada, N., Oguri, M., Morokuma, T., et al. 2006, ApJL, 653, 97

Inada, N., Oguri, M., Pindor, B., et al. 2003, Natur, 426, 810

Kaspi, S., Smith, P. S., Netzer, H., et al. 2000, ApJ, 533, 631

Konigl, A., \& Kartje, J. F. 1994, ApJ, 434, 446

Krolik, J. H., \& Kriss, G. A. 2001, ApJ, 561, 684

Lopez, S., Reimers, D., Gregg, M. D., et al. 2005, ApJ, 626, 767

Lu, L., Sargent, W. L. W., Barlow, T. A., Churchill, C. W., \& Vogt, S. S. 1996, ApJS, 107, 475

Marziani, P., Sulentic, J. W., Dultzin-Hacyan, D., Calvani, M., \& Moles, M. 1996, ApJS, 104, 37

McLure, R. J., \& Dunlop, J. S. 2004, MNRAS, 352, 1390

Misawa, T., Charlton, J. C., Eracleous, M., et al. 2007a, ApJS, 171, 1

Misawa, T., Eracleous, M., Charlton, J. C., \& Kashikawa, N. 2007b, ApJ, 660,152

Misawa, T., Eracleous, M., Charlton, J. C., \& Tajitsu, A. 2005, ApJ, 629,115

Misawa, T., Tytler, D., Iye, M., et al. 2004, AJ, 128, 2954

Murray, N., Chiang, J., Grossman, S. A., \& Voit, G. M. 1995, ApJ, 451,498

Narayanan, D., Hamann, F., Barlow, T., et al. 2004, ApJ, 601, 715

Nestor, D., Hamann, F., \& Rodriguez Hidalgo, P. 2008, MNRAS, 386,2055

Netzer, H., Lira, P., Trakhtenbrot, B., Shemmer, O., \& Cury, I. 2007, ApJ, 671,1256

Oguri, M., Ofek, E. O., Inada, N., et al. 2008, ApJL, 676, 1

Oguri, M., Schrabback, T., Jullo, E., et al. 2012, arXiv:1209.0458

Ohsuga, K., Mori, M., Nakamoto, T., \& Mineshige, S. 2005, ApJ, 628, 368

Ota, N., Oguri, M., Dai, X., et al. 2012, ApJ, 758, 26

Penton, S. V., Stocke, J. T., \& Shull, J. M. 2004, ApJS, 152, 29

Perry, J. J., Burbidge, E. M., \& Burbidge, G. R. 1978, PASP, 90, 337

Petitjean, P., \& Bergeron, J. 1994, A\&A, 283, 759

Pichon, C., Scannapieco, E., Aracil, B., et al. 2003, ApJL, 597, 97

Proga, D., Stone, J. M., \& Drew, J. E. 1998, MNRAS, 295, 595

Proga, D., Stone, J. M., \& Drew, J. E. 1999, MNRAS, 310, 476

Proga, D., Stone, J. M., \& Kallman, T. R. 2000, ApJ, 543, 686

Rauch, M., Sargent, W. L. W., \& Barlow, T. A. 1999, ApJ, 515, 500

Reichard, T. A., Richards, G. T., Schneider, D. P., et al. 2003, AJ, 125, 1711

Sargent, W. L. W., Young, P. J., Boksenberg, A., \& Tytler, D. 1980, ApJS, 42,41

Shen, Y., Richards, G. T., Strauss, M. A., et al. 2011, ApJS, 194, 45

Sprayberry, D., \& Foltz, C. B. 1992, ApJ, 390, 39

Springel, V., Di Matteo, T., \& Hernquist, L. 2005, ApJL, 620, 79

Stocke, J. T., Morris, S. L., Weymann, R. J., \& Foltz, C. B. 1992, ApJ, 396, 487

Tytler, D., \& Fan, X.-M. 1992, ApJS, 79, 1

Vestergaard, M. 2003, ApJ, 599, 116 
Voit, G. M., Weymann, R. J., \& Korista, K. T. 1993, ApJ, 413, 95

Wampler, E. J., Chugai, N. N., \& Petitjean, P. 1995, ApJ, 443, 586

Weymann, R. J., Carswell, R. F., \& Smith, M. G. 1981, ARA\&A, 19,41

Weymann, R. J., Morris, S. L., Foltz, C. B., \& Hewett, P. C. 1991, ApJ, 373,23
Weymann, R. J., Williams, R. E., Peterson, B. M., \& Turnshek, D. A. 1979, ApJ 234,33

Wise, J. H., Eracleous, M., Charlton, J. C., \& Ganguly, R. 2004, ApJ, 613, 20

Yamamoto, T. M., \& Vansevičius, V. 1999, PASJ, 51, 405

York, D. G., Adelman, J., Anderson, J. E., Jr., et al. 2000, AJ, 120, 1579

Young, P., Sargent, W. L. W., \& Boksenberg, A. 1982, ApJS, 48, 455 